\title{
Platelets: inflammatory effector cells in the conflagration of cystic fibrosis lung disease
}

\author{
Guy A. Zimmerman
}

Division of Pulmonary and Critical Care Medicine, Department of Internal Medicine and Program in Molecular Medicine, University of Utah School of Medicine, Salt Lake City, Utah, USA.

\begin{abstract}
Cystic fibrosis (CF) is a multisystem disorder, but progressive inflammatory lung disease causes the greatest burden of morbidity and death. Recent translational and mechanistic studies of samples from patients, and observations in animal models, indicate that platelets may drive lung injury and contribute to dysregulated host defense in CF lung disease. In this issue of the $J C l$, Ortiz-Muñoz and Yu et al. explored the role that the cystic fibrosis transmembrane conductance regulator (CFTR) plays in platelet-related inflammation. The authors used mouse and human model systems to show that CFTR dysfunction in platelets increased calcium entry though the transient receptor potential cation channel 6 (TRPC6), causing hyperactivation and consequent experimental lung inflammation. The study persuasively suggests that platelets are critical thromboinflammatory effector cells in CF lung disease. In the context of platelet-related organ injury seen in a variety of other diseases and syndromes, platelets may also contribute to nonpulmonary manifestations and comorbidities of CF.
\end{abstract}

\section{Dysregulated lung inflammation}

Inflammation is a process of seemingly infinite complexity. The inflammatory environment of the lung in cystic fibrosis (CF) is a dramatic example. The gene for the cystic fibrosis transmembrane conductance regulator (CFTR in humans, cftr in mice) is mutated in patients with $\mathrm{CF}$, causing a relentless temporal cascade of pathologic events beginning in infancy and culminating in unregulated neutrophilic inflammation, an injurious proinflammatory milieu, chronic with superimposed episodic bacterial infection, mucus obstruction of airways, bronchiolitis obliterans, and bronchiectasis $(1,2)$. Although there is heterogeneity in $\mathrm{CF}$, in many patients this maladaptive sequence leads to respiratory failure and premature death absent the option of lung transplan- tation (2). The key mechanisms involved in injurious airway inflammation, somehow triggered by deficiency or dysfunction of the cystic fibrosis transmembrane conductance regulator protein (CFTR in humans, cftr in mice) in critical cells, and in the ubiquitous microbial colonization and infection of the lung are ongoing conundrums $(1,2)$. Informative discoveries relevant to the dysregulated lung inflammation and persistent infection in CF could provide avenues for new therapeutic intervention in this pernicious genetic disorder.

Platelets, the cardinal cells of hemostasis and thrombosis, are not recognized as inflammatory effector cells by most clinicians and investigators. Nevertheless, they are $(3,4)$. Recent observations demonstrate this point in the context of $\mathrm{CF}$, providing evidence that CFTR influences activation and proinflammatory

Related Article: p. 2041

Conflict of interest: GAZ is a coinventor on patent applications, including the most recent US patent publication US 2019/0307865 A1, titled "Methods for treatment of and prophylaxis against inflammatory disorders."

Copyright: ( 2020, American Society for Clinical Investigation.

Reference information: J Clin Invest. 2020;130(4):1632-1634. https://doi.org/10.1172/JCI135949.

responses of human platelets and that platelets contribute to lung inflammation in mouse models relevant to CF (5).

\section{Aberrant activation of CF platelets}

Ortiz-Muñoz, $\mathrm{Yu}$, and coworkers in Mark Looney's laboratory in San Francisco have made substantive observations regarding platelets in the lung. In this issue of the JCI they found that platelets isolated from subjects with CF (outpatients with substantial lung involvement not in exacerbation) and platelets from healthy controls treated in vitro with a CFTR inhibitor responded to thrombin stimulation with enhanced translocation of $\mathrm{P}$ selectin $(\mathrm{CD} 62 \mathrm{P})$ and signaling of integrin $\alpha_{11 b} \beta_{3}$ (glycoprotein IIb/IIIa) (5). These are physiologically relevant platelet activation events that mediate thromboinflammatory effector functions (3). Previously published findings also indicate that circulating platelets have augmented activation responses in CF (6-9). The Ortiz-Muñoz and Yu et al. study provided a potential mechanism for at least some aspects of exaggerated, aberrant activation of CF platelets: inhibitor experiments demonstrated that CFTR influences calcium flux in platelets regulated by a second plasma membrane channel, transient receptor potential cation channel 6 (TRPC6). Additional analysis of platelets from genetically altered mice was consistent with these results.

Together, the findings support the conclusion that dysfunction or deficiency of CFTR on platelets causes increased calcium entry and consequent hyperactivation. Pharmacologic modulation of TRPC6 normalized calcium entry and activation of human platelets with dysfunctional CFTR. Another interesting observation was that therapeutic administration of a modulator of CFTR (lumacaftor/ivacaftor) in a small subset of the CF patients partially blunted enhanced platelet activation. This suggested that platelets may be accessible reporters 


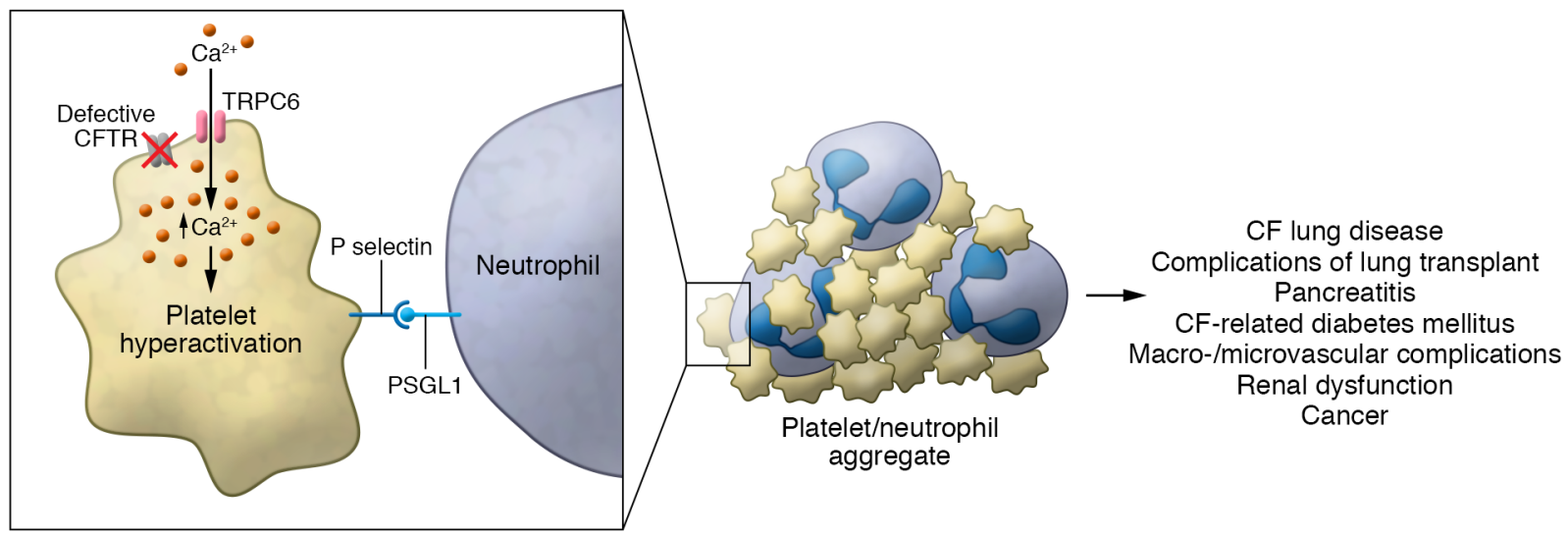

Figure 1. Model for platelets as inflammatory effector cells in the conflagration of cystic fibrosis lung disease. Defective CFTR induces increased calcium influx through TRPC6, resulting in hyperactivation of platelets when they are stimulated by inflammatory and prothrombotic agonists. Consequent binding of P selectin to PSGL-1 on neutrophils and other leukocytes contributes to thromboinflammatory injury in multiple organs and systems relevant to CF. Other effector responses of activated platelets, including adhesive interactions via integrin $\alpha_{\| 1} \beta_{3}$ and signaling of NET formation, may also contribute to CF complications and comorbidities.

of CFTR modulator efficacy and in new CF therapeutic development (5).

\section{Drivers of injurious lung inflammation in CF}

In parallel, mouse models revealed that platelets deficient in cftr contribute to lung inflammation. Ortiz-Muñoz and Yu et al. generated mice with lineage-specific conditional deletion of $c f t r$ selectively in platelets and, for comparison, in myeloid leukocytes and neutrophils, and obtained mice that are globally deficient in $c f t r\left(c f t r^{--}\right)$in most cells, including platelets. They also used wild-type (WT) mice and mice from parent strains employed for conditional $c f t r$ deletions as controls. Platelets deficient in $c f t r$ responded to thrombin with enhanced activation (P selectin translocation) compared with controls, a pattern similar to that of circulating platelets from patients with CF. When challenged with intratracheal lipopolysaccharide (LPS) or Pseudomonas aeruginosa, mice selectively deficient in platelet $c f t r$ and $c f t r-$ mice showed excessive alveolar inflammation and permeability compared with control animals, indicated by increased total leukocytes, neutrophils, and protein in bronchoalveolar lavage (BAL) samples. Pseudomonas aeruginosa is a predominant pathogen in the complex microbiome of CF (2), establishing the relevance of this model. The LPS model was identified as a reductionist experimental tool for these studies (5), but there is evidence - not unexpected - that there is a burden of LPS in the CF lung and circulation (10). Of note, both human and mouse platelets express toll-like receptor 4 in their immune repertoire, and sense and respond to LPS $(3,11)$.

Markers of platelet activation were increased in BAL and blood samples from cftr-deficient mice in both the LPS and Pseudomonas models. Evidence for platelet-leukocyte interaction was a particularly important feature. BAL and blood platelet-neutrophil and platelet-monocyte aggregates were more abundant in mice with platelet-selective $c f t r$ deficiency than in controls in both challenge models. Platelet-leukocyte aggregates form via a P selectin-dependent mechanism in response to platelet activation (reviewed in ref. 12) and have been detected in the blood of patients with CF $(7,9)$ and in real time in microvessels of the inflamed mouse lung (13). Platelet-leukocyte interactions of this nature induce proinflammatory signaling and mediator synthesis $(3,12,14)$, and could drive injurious lung inflammation in CF. In addition, markers of neutrophil extracellular trap (NET) formation were increased in samples from $\mathrm{cftr}^{-1-}$ mice compared with samples from WT animals and heterozygotes. NET formation is thought to be a mechanism of lung defense and, conversely, pulmonary and extrapulmonary tissue injury $(3,11,15)$. Platelet-neutrophil interaction can trigger NETs $(11,16)$, but this relationship was not specifically examined in the current study. NETs can also be generated by other mechanisms, includ- ing direct interaction of neutrophils with pathogens (3).

Another interesting finding was that bacterial colony counts in the lungs of $\mathrm{cftr}^{-1}$ mice and mice with selective platelet $c f t r$ deficiency were higher than those in the lungs of control animals challenged with intratracheal Pseudomonas (5). Although mouse models are imperfect surrogates for CF lung disease in humans $(1,5)$, this is strikingly similar to the dramatic dysregulation of host defense in the lungs of affected patients with $\mathrm{CF}$ : persistent pathogen burden in the presence of exuberant inflammation that is ineffective in clearing the invading microbes but - in humans - causes devastating collateral damage $(1,2)$. It will ultimately be interesting to assess platelet behavior in the context of other infections of particular importance to CF, such as Burkholderia (2). Genetic deletion of trpc6 in $c \mathrm{ftr}^{-/}$mice restored bacterial clearance and reduced indices of inflammation and heightened alveolar permeability in the Pseudomonas pneumonia model (5). In addition, alveolar inflammation, permeability, and indices of platelet activation and NET formation were reversed in $c f t r^{--}, \operatorname{trpc} 6^{-/-}$double knockouts in the LPS model (5), with levels similar to those in littermate control mice, a result consistent with the mechanistic studies of platelet calcium entry and activation profiled above. In aggregate, the mouse and in vitro experiments suggest that deficiency or dysfunction of platelet CFTR and resulting aberrant TRPC6- 
dependent platelet activation may contribute to impaired bacterial clearance and injurious lung inflammation in $\mathrm{CF}$. The authors also suggested that platelets and TRPC6 are targets for treatment in CF lung disease (5). Antiplatelet therapy for this disorder was raised previously $(7,8)$. A caveat, however, is that traditional antiplatelet drugs may not inhibit all platelet inflammatory responses $(3,17)$.

\section{Traditional and nontraditional platelet activities}

Platelets have an amicus and adversary relationship with the lung - defensive and damaging, yin and yang - in acute injury syndromes (17). A paradoxical relationship of this nature may also exist in CF. Traditional hemostatic effector functions of platelets are requisite in control of pulmonary hemorrhage, a dangerous CF complication (18), and platelets may have critical airway defense, antibacterial, and repair activities $(3,12)$ as well. Yet platelets may also have a dark side in CF, and contribute to lung pathology. Venous thromboembolism and central venous catheter thrombosis are vexing problems in CF $(18,19)$, likely in part because of the complicated links between hemostasis and inflammation $(3,12)$. It is unknown if increased activation responses of circulating platelets (5-9) contribute to these thrombotic complications, but if so this could be another rationale for antiplatelet therapy. And the observations by Ortiz-Muñoz and Yu et al. indicate that CFTR-deficient platelets can drive inflammatory lung injury, a central component of CF pulmonary disease (1, 2 ), at least in the experimental setting (5).

Interesting issues related to recently discovered, nontraditional activities of platelets $(3,12,17)$ may be relevant to the pathology of $\mathrm{CF}$, but have not been investigated. For example, platelets have an extensive transcriptome that is dynamically altered in disease $(3,20)$. It would be useful to know if the platelet transcriptome changes in $\mathrm{CF}$ and, if so, whether there are consequent changes in the platelet proteome that influence lung inflammation. Activated platelets release, and in some cases synthesize, inflammatory mediators, including IL-1 $\beta(3,12)$, an inflammasome-processed cytokine that may be particularly important (10) in the toxic microenvironment (2) of the CF lung. Assessment of the contributions of plateletderived cytokines, chemokines, and lipids to this toxic milieu would be informative.

Studies of other diseases and disease models (12) indicate that thromboinflammatory functions of activated platelets may contribute to complications and comorbidities of CF $(2,18,19)$ in addition to CF lung disease (5), with platelet-neutrophil and other platelet-leukocyte interactions as possible important mechanisms $(3,12)$. Some CF comorbidities, such as cancers and CF diabetes-related vascular involvement, are increasing in frequency as therapy for this disease improves and patient life span increases $(2,18)$. Contributions of platelets and megakaryocytes, their parent cells $(3,12)$, to the devastating conflagration of CF are probably not limited to the lung (Figure 1).

\section{Acknowledgments}

The author was supported by National Heart, Lung and Blood Institute award R37HL044525 while work referenced in this commentary was accomplished. Kendra Richardson provided invaluable assistance in preparation of the manuscript.

Address correspondence to: Guy A. Zimmerman, 4220 Eccles Institute of Human Genetics, University of Utah Medical Center, Salt Lake City, Utah 84112, USA. Phone: 801.585.0950; Email: guy.zimmerman@ u2m2.utah.edu.

1. Stoltz DA, Meyerholz DK, Welsh MJ. Origins of cystic fibrosis lung disease. $N$ Engl J Med. 2015;372(4):351-362.

2. Elborn JS. Cystic fibrosis. Lancet. 2016;388(10059):2519-2531.

3. Middleton EA, Weyrich AS, Zimmerman GA. Platelets in pulmonary immune responses and inflammatory lung diseases. Physiol Rev. 2016;96(4):1211-1259.

4. Morrell CN, Pariser DN, Hilt ZT, Vega Ocasio D. The platelet Napoleon complex-small cells, but big immune regulatory functions. Annu Rev Immunol. 2019;37:125-144.
5. Ortiz-Muñoz G, et al. Cystic fibrosis transmembrane conductance regulator dysfunction in platelets drives lung hyperinflammation. JClin Invest. 2020;130(4):2041-2053.

6. Ciabattoni $\mathrm{G}$, et al. In vivo lipid peroxidation and platelet activation in cystic fibrosis. Am J Respir Crit Care Med. 2000;162(4 Pt 1):1195-1201.

7. O'Sullivan BP, et al. Platelet activation in cystic fibrosis. Blood. 2005;105(12):4635-4641.

8. O'Sullivan BP, Michelson AD. The inflammatory role of platelets in cystic fibrosis. Am J Respir Crit Care Med. 2006;173(5):483-490.

9. Mattoscio D, et al. Cystic fibrosis transmembrane conductance regulator (CFTR) expression in human platelets: impact on mediators and mechanisms of the inflammatory response. FASEB J. 2010;24(10):3970-3980.

10. McElvaney OJ, et al. Specific inhibition of the NLRP3 inflammasome as an antiinflammatory strategy in cystic fibrosis. Am J Respir Crit Care Med. 2019;200(11):1381-1391.

11. Clark SR, et al. Platelet TLR4 activates neutrophil extracellular traps to ensnare bacteria in septic blood. Nat Med. 2007;13(4):463-469.

12. Vieira-de-Abreu A, Rondina MT, Weyrich AS, Zimmerman GA. Inflammation. In: Michelson AD, ed. Platelets, 3rd ed. Amsterdam, Netherlands: Elsevier/North Holland;2013:733-766.

13. Ortiz-Muñoz G, Mallavia B, Bins A, Headley M, Krummel MF, Looney MR. Aspirin-triggered 15-epi-lipoxin A4 regulates neutrophil-platelet aggregation and attenuates acute lung injury in mice. Blood. 2014;124(17):2625-2634.

14. Weyrich AS, McIntyre TM, McEver RP, Prescott SM, Zimmerman GA. Monocyte tethering by P-selectin regulates monocyte chemotactic protein-1 and tumor necrosis factor-alpha secretion. Signal integration and NF-kappa B translocation. J Clin Invest. 1995;95(5):2297-2303.

15. Lefrançais E, Mallavia B, Zhuo H, Calfee CS, Looney MR. Maladaptive role of neutrophil extracellular traps in pathogen-induced lung injury. JCI Insight. 2018;3(3):98178.

16. Caudrillier A, et al. Platelets induce neutrophil extracellular traps in transfusion-related acute lung injury. J Clin Invest. 2012;122(7):2661-2671.

17. Middleton EA, Rondina MT, Schwertz H, Zimmerman GA. Amicus or adversary revisited: platelets in acute lung injury and acute respiratory distress syndrome. Am J Respir Cell Mol Biol. 2018;59(1):18-35.

18. Plant BJ, Goss CH, Plant WD, Bell SC. Management of comorbidities in older patients with cystic fibrosis. Lancet Respir Med. 2013;1(2):164-174.

19. Knight-Perry J, Branchford BR, Thornhill D, Martiniano SL, Sagel SD, Wang M. Venous thromboembolism in children with cystic fibrosis: Retrospective incidence and intrapopulation risk factors. Thromb Res. 2017;158:161-166.

20. Schubert S, Weyrich AS, Rowley JW. A tour through the transcriptional landscape of platelets. Blood. 2014;124(4):493-502. 